\title{
Correlation of health-related quality of life with other disease severity indices in Indian chronic obstructive pulmonary disease patients
}

This article was published in the following Dove Press journal:

International Journal of COPD

30 April 2012

Number of times this article has been viewed

\author{
Seema Aziyakath Shavro' \\ Punitha Ezhilarasu' \\ Jebamani Augustine' \\ Joel J Bechtel ${ }^{2}$ \\ Devasahayam J \\ Christopher ${ }^{3}$ \\ 'College of Nursing, Christian \\ Medical College, Vellore, South \\ India; ${ }^{2}$ Pulmonologist and Critical \\ Care Physician, Grand Junction, $\mathrm{CO}$, \\ ${ }^{3}$ Department of Pulmonary Medicine, \\ Christian Medical College, Vellore, \\ South India
}

Background: Improvement in quality of life (QOL) has become a focus for the management of incurable chronic diseases, including chronic obstructive pulmonary disease (COPD). This study investigates factors influencing the QOL of patients with COPD in India.

Methods: Seventy-three consecutive COPD patients visiting an outpatient pulmonary clinic underwent health-related QOL (HRQOL) assessment using the World Health Organization's QOL abbreviated questionnaire and St George's Respiratory Questionnaire (SGRQ). Symptom severity and grade of dyspnea were estimated by the Chronic Lung Disease Severity Index (CLD) and Medical Research Council assessments, and patient demographic data were collected. Spirometry and 6-minute walk tests were performed to assess lung function and functional status.

Results: Patients with COPD showed significantly reduced HRQOL when measured by the World Health Organization's QOL abbreviated questionnaire and the SGRQ. CLD estimate for severity of lung disease $(P<0.001)$, Medical Research Council assessment for dyspnea $(P<0.01)$, and duration of illness $(P<0.05)$ showed close correlation with HRQOL. Worsening forced expiratory volume in 1 second and 6-minute walk test results closely correlated with poorer HRQOL $(P<0.01)$. No association between QOL and age, quantum of smoking, education, comorbid illnesses, or occupational exposure was found.

Conclusion: This study showed that Indian patients with COPD had reduced HRQOL. Longer disease duration, patient perception of disease severity, and worsening dyspnea impacted negatively on HRQOL.

Keywords: quality of life, COPD, disease severity, India, questionnaire

\section{Introduction}

The Global Initiative for Chronic Obstructive Lung Diseases (GOLD) 2001 defined chronic obstructive pulmonary disease (COPD) as a disease state characterized by chronic airflow obstruction that is not fully reversible. This airflow limitation is usually both progressive and associated with an abnormal inflammatory response of the lungs to noxious particles or gases. ${ }^{1}$ COPD progressively reduces breathing capacity and impairs patients' ability to carry out activities of daily living, thereby adversely affecting health-related quality of life (HRQOL). A World Health Organization (WHO)/World Bank study estimated the prevalence of COPD per 1000 population in India as 4.38 in males and 3.44 in females. ${ }^{2}$ With the increasing prevalence of smoking in developing countries, it is projected that COPD-related mortality and morbidity will dramatically impact Asian and African countries. ${ }^{3}$ COPD is an irreversible and progressive disease, and hence medical and surgical care results in very little improvement of lung function 
or survival of these individuals, aside from the benefits seen with use of long-term oxygen treatment. Patients with COPD experience reduced HRQOL. Some factors such as depression, poor mobility, dyspnea, and inadequate social support adversely affect HRQOL, and these factors may be more amenable to treatment than treating only lung function impairment. Patient-focused HRQOL studies will provide the health care personnel with specific information regarding the problems that these patients experience and will thereby enable them to develop interventions directed toward improving their care.

There is a paucity of published literature regarding factors affecting the QOL of COPD patients in India. The present study aimed at determining the relationship between QOL and selected demographic and physiologic variables and symptom severity scores of patients with COPD.

\section{Materials and methods Study design}

Patients visiting their pulmonologist for initial consultation for dyspnea or follow-up outpatient visits for COPD were invited to participate in this study. Basic demographic data and the generic WHOQOL abbreviated questionnaire (WHOQOL-BREF) ${ }^{4}$ were completed on the first visit, and 1 week later the disease-specific St George's Respiratory Questionnaire (SGRQ) ${ }^{5}$ clinical and physiologic data were gathered. This prospective study was approved by the Christian Medical College, Vellore, South India, and the hospital's institutional review board.

\section{Patient population}

Seventy-three consecutive COPD patients were enrolled over a 2-month period in this study, which was performed at the pulmonary medicine outpatient clinic at Christian Medical College and hospital, a 2100-bed tertiary referral hospital and medical school in Tamilnadu, South India. Inclusion criteria for patient participation consisted of age over 40 years and irreversible airway obstruction on spirometry. Fifteen subjects were ineligible due to their inability to perform a 6-minute walk (nine), refusal to participate (three), additional coexisting lung disease (two), and flare-up of COPD (one).

\section{Testing methods}

Spirometry was performed using a pulmonary function testing system (MasterScreen PFT ${ }^{\circledR}$; Jaeger, Höchberg, Germany). Pre- and postbronchodilator (salbutamol $400 \mathrm{mcg}$ ) maneuvers were done by qualified respiratory therapists following American Thoracic Society standards. ${ }^{6}$ COPD was documented by airflow obstruction showing forced expiratory volume in 1 second $\left(\mathrm{FEV}_{1}\right)$ /forced vital capacity (FVC) $<70 \%$ and less than 200 cc increase in $\mathrm{FEV}_{1}$ post bronchodilator. Disease severity was classified according to GOLD criteria. A 6-minute walk test was performed in a 30 -meter corridor.

HRQOL was measured using two questionnaires: the generic WHOQOL-BREF ${ }^{4}$ and the disease-specific SGRQ. ${ }^{5}$

The WHOQOL-BREF has 26 questions, of which the first two yield information about the individual's overall perception of QOL and overall perception of health. The remaining questions assess QOL in four domains: physical, psychological, social relationships, and environmental. It is a positively scored questionnaire, with the scores of the negatively worded questions being reversed for total score calculation. Higher scores indicate better QOL. The final scores were interpreted as follows: $\geq 75 \%=$ good QOL, $50 \%-74 \%=$ fair QOL, and $<50 \%=$ poor QOL.

The SGRQ is a standardized questionnaire used to assess the effect of respiratory diseases on QOL. It is divided into three sections: "symptoms" deals with the frequency and severity of respiratory manifestations, "activity" relates to activities that cause or are limited by breathlessness, and "impacts" covers the aspects of social function and psychosocial disturbances that result from respiratory diseases.

Scores were expressed as percentage of overall impairment, where 100 represents worst possible health and 0 indicates best possible health status. Higher status on SGRQ indicates poorer QOL.

The Chronic Lung Disease Severity Index (CLD) ${ }^{7}$ was used to quantify respiratory symptoms. This short six-item questionnaire evaluated dyspnea, wheezing, and productive cough. Raw scores were added and transformed to a scale of 0 (normal) to 100 (most severe). Dyspnea was assessed by the Medical Research Council (MRC) five-point scale for breathlessness.

The demographic variables, such as education, occupation, and smoking status, were analyzed with the mean QOL scores using the Kruskal-Wallis test, and the relationship between HRQOL scores and continuous variables were assessed using Spearman's correlation coefficient $(r)$. A $P$-value of $<0.05$ was considered statistically significant.

\section{Results}

Patient demographic results show a mean age of 62 years. Fifty-four were male (93\%), 56 were married (97\%), 
Table I Demographic characteristics of 58 subjects with chronic obstructive pulmonary disease

\begin{tabular}{ll}
\hline Characteristic & Value \\
\hline Mean age \pm standard deviation (years) & $62.4 \pm 7.83$ \\
Sex & \\
Male & $54(93 \%)$ \\
Female & $4(7 \%)$ \\
Marital status & \\
Unmarried & 0 \\
Married & $56(97 \%)$ \\
Widowed/widower & $2(3 \%)$ \\
Divorced & 0 \\
Education & \\
Illiterate & $9(15 \%)$ \\
Primary & $7(12 \%)$ \\
Secondary & $28(48 \%)$ \\
Graduate and above & $14(24 \%)$ \\
Smoking status & \\
Never smoked & $10(17 \%)$ \\
Quit smoking & $39(67 \%)$ \\
Current smoker & $9(16 \%)$ \\
Mean pack-years of tobacco smoked & $32 \pm 34.55$ \\
( \pm standard deviation) & \\
\hline
\end{tabular}

49 completed some formal schooling (86\%), and 48 were current or ex-smokers (83\%), with an average 32 pack-year smoking history (Table 1).

Clinical characteristics showed a mean duration of illness of 6 years; a mean CLD index of 55, indicating moderately

Table 2 Clinical characteristics of the 58 study subjects

\begin{tabular}{ll}
\hline Clinical variables & Values \\
\hline Mean duration of illness \pm standard deviation (years) & $6.07 \pm 6.06$ \\
Mean CLD Severity Index & $55.03 \pm 14.19$ \\
Comorbid condition (number of patients) & \\
Nil & $20(34 \%)$ \\
Gastric disease & $12(21 \%)$ \\
Hypertension & $11(19 \%)$ \\
Diabetes mellitus & $10(17 \%)$ \\
Cataract & $9(16 \%)$ \\
Heart disease & $9(16 \%)$ \\
Prostatic disease & $4(7 \%)$ \\
Osteoarthritis/rheumatoid arthritis & $4(7 \%)$ \\
Miscellaneous & $6(10 \%)$ \\
Number of patients with comorbid conditions & \\
0 & $20(34 \%)$ \\
I & $17(29 \%)$ \\
2 & $14(24 \%)$ \\
$\geq 3$ & $7(12 \%)$ \\
Mean dyspnea score (MRC grade ) \pm standard deviation & $2.6 \pm 1.35$ \\
Long-term oxygen therapy & \\
Yes & $0 \%$ \\
No & $58 \%(100 \%)$ \\
\hline Ab &
\end{tabular}

Abbreviations: CLD, Chronic Lung Disease Severity Index; MRC, Medical Research Council.
Table 3 Spirometry and 6-minute walk results of the study subjects

\begin{tabular}{ll}
\hline Physiological variables & Values \\
\hline Disease severity (\%) & \\
Stage I (FEV,$\geq 80 \%$ of the predicted value) & $\mathrm{I}(2 \%)$ \\
Stage IIA (FEV , 50\%-79\% of the predicted value) & $25(43 \%)$ \\
Stage IIB (FEV, 30\%-49\% of the predicted value) & $21(36 \%)$ \\
Stage III (FEV, $<30 \%$ of the predicted value) & $1 \mathrm{II}(19 \%)$ \\
Mean FEV $\%$ predicted \pm standard deviation & $47.6 \pm 18.38$ \\
Mean 6-minute distance walked \pm standard deviation & $359.2 \pm 92.52$ \\
(meters) & \\
\hline
\end{tabular}

Abbreviation: $\mathrm{FEV}_{1}$, forced expiratory volume in I second.

severe disease; a dyspnea severity score of 2.6 , indicating moderately severe dyspnea; and comorbid illness found in $65 \%$ (Table 2).

Spirometry showed that one subject was GOLD (2001) Stage I, 46 were Stage II, and eleven were Stage III. Mean 6-minute walk was 359 meters (Table 3).

WHOQOL-BREF results showed that 14 patients rated QOL to be good, 31 fair, and 13 poor (Tables 4 and 5). When separate domains were analyzed, the worse the overall QOL, the worse the physical symptoms, whereas trends with psychological and social scores suggested that these remained relatively high despite advanced disease. The SGRQ scores likewise showed significant impairment in all three domain measures, symptoms, activity, and impacts, when compared with reference values ${ }^{5}$ (Table 4).

No association was identified between QOL and the demographic variables age, education, occupation exposure, smoking status, or pack-years of tobacco smoked (Table 6).

Duration of illness showed a significant correlation with the WHOQOL-BREF questionnaire $(P<0.05)$, indicating that as duration of illness increased, the QOL

Table 4 Mean health-related quality of life scores

\begin{tabular}{llll}
\hline Quality of life & Mean & $\begin{array}{l}\text { Standard } \\
\text { deviation }\end{array}$ & Range \\
\hline WHOQOL-BREF & & & \\
Physical domain & 51.60 & 23.47 & $6-94$ \\
Social domain & 62.03 & 27.26 & $6-100$ \\
Psychological domain & 71.78 & 19.56 & $25-100$ \\
Environmental domain & 64.29 & 14.23 & $30-94$ \\
Total & 62.50 & 15.63 & $28-94$ \\
SGRQ & & & \\
Symptoms & 41.64 & 20.99 & $0-93$ \\
Activity & 62.57 & 24.61 & $0-100$ \\
Impacts & 39.21 & 23.52 & $0-92$ \\
Total & 46.69 & 21.61 & $0-88$ \\
\hline
\end{tabular}

Abbreviations: SGRQ, St George's Respiratory Questionnaire; WHOQOL-BREF, World Health Organization's quality of life abbreviated questionnaire. 
Table 5 WHOQOL-BREF results showing number of patients with chronic obstructive pulmonary disease by total, domain, and mean score

\begin{tabular}{lllll}
\hline & $\begin{array}{l}\text { Good } \\
(>\mathbf{7 5 )}\end{array}$ & $\begin{array}{l}\text { Fair } \\
(\mathbf{5 0 - 7 5 )}\end{array}$ & $\begin{array}{l}\text { Poor } \\
(<\mathbf{5 0})\end{array}$ & $\begin{array}{l}\text { Mean/ } \\
\text { standard deviation }\end{array}$ \\
\hline Patients, total & 14 & 31 & 13 & $62.5 \pm 15.6$ \\
Patients by domain & & & \\
Physical & 13 & 18 & 27 & $51.6 \pm 23.5$ \\
Social & 25 & 15 & 18 & $62.0 \pm 27.3$ \\
Psychological & 38 & 13 & 7 & $71.8 \pm 19.6$ \\
Environmental & 16 & 34 & 8 & $64.3 \pm 14.2$ \\
\hline
\end{tabular}

Abbreviation: WHOQOL-BREF, World Health Organization's quality of life abbreviated questionnaire.

worsened (Table 8 ). The CLD severity index correlated significantly with both the WHOQOL-BREF $(P<0.05)$ and SGRQ $(P<0.001)$, suggesting that the greater the severity of symptoms, the poorer the QOL. The MRC dyspnea grade also showed significant correlation $(P<0.001)$ with both QOL questionnaires. No association was found between comorbid illness and QOL (Table 7).

Lung function as measured by $\mathrm{FEV}_{1}$ showed significant correlations with both WHOQOL-BREF $(P<0.001)$ and SGRQ $(P<0.001)$ QOL assessments, indicating that poorer lung function was associated with poorer QOL (Table 8). Likewise, the $\mathrm{FEV}_{1} / \mathrm{FVC}$ showed correlation to a lesser degree with the WHOQOL-BREF $(P<0.05)$ and SGRQ $(P<0.05)$. The 6-minute walk, a functional assessment for lung disease, showed significant correlation with WHOQOLBREF $(P=0.001)$ and SGRQ $(P=0.005)$ (Table 8$)$.

\section{Discussion}

This study showed that 58 consecutive Indian patients with COPD had significantly impaired QOL when measured by the generic questionnaire WHOQOL-BREF and the disease-specific questionnaire SGRQ. When examining specific domains, all showed impairment; however, no clear trends were apparent. The social and psychological domains on the WHO questionnaire were rated less impaired; however, the "impacts" domain (social and psychosocial) on the SGRQ appeared more impaired. Jones and Spencer ${ }^{5}$ suggested that a four-unit difference in SGRQ score between groups of patients indicated a clinically significant difference. Components adversely affecting HRQOL include frequency and severity of respiratory symptoms, breathlessness causing limitations of activity, poor social functions, and psychological disturbances. By concentrating investigation and treatment on these selected components, it is hoped that patients with COPD might lead fuller and more satisfying lives. Antidepressants are helpful for psychological disturbances, bronchodilators and narcotics can help dyspnea, and pulmonary rehabilitation programs, which are a rarity in India, show functional improvement of the 6-minute walk. Improvements in peer groups and family support may benefit patients by providing more social structure.

Measures of perceived severity of illness (CLD) and dyspnea (MRC) likewise showed tight correlation with QOL measurements in the study, similar to prior studies. ${ }^{8,9} \mathrm{QOL}$ is defined by the WHO as an individual's perceptions of their position in life in the context of the culture and value systems in which they live. This study shows that in India, COPD patients suffer similar reductions in QOL as do COPD patients from other countries, as reported previously. ${ }^{10,11}$

Lung functions as measured by $\mathrm{FEV}_{1}$ and classified as per GOLD criteria showed in this study that the lower the $\mathrm{FEV}_{1}$, the lower the patient's QOL. This association of $\mathrm{FEV}_{1}$ and QOL confirms findings from previous studies ${ }^{10,12}$ and was anticipated. With worsening lung function, normal activity is limited or curtailed. All QOL domains appeared

Table 6 Correlation of SGRQ and WHOQOL-BREF scores with demographic variables

\begin{tabular}{|c|c|c|c|c|c|}
\hline \multirow[t]{2}{*}{ Variable } & \multicolumn{4}{|l|}{ SGRQ } & \multirow[t]{2}{*}{ WHOQOL-BREF } \\
\hline & Symptom & Activity & Impacts & Total & \\
\hline Age,$r$-value & 0.079 & 0.175 & 0.035 & 0.056 & -0.135 \\
\hline Pack-years of tobacco smoked ${ }^{\mathrm{a}}, r$-value & 0.16 & 0.19 & 0.19 & 0.18 & 0.03 \\
\hline \multicolumn{6}{|c|}{ Education ${ }^{\mathrm{b}}$, mean (standard deviation) } \\
\hline Illiterate & $43.06(20.9)$ & $63.44(28.2)$ & $39.38(21.2)$ & $47.38(20.8)$ & $59.88(16.3)$ \\
\hline Secondary school & $44.32(21.9)$ & $63.25(21.9)$ & $41.68(23.1)$ & $48.64(20.9)$ & $61.21(14.9)$ \\
\hline Graduate/professional & 34.64 (19.1) & $60.21(27.2)$ & 34.07 (27.5) & $42.00(24.7)$ & 68.07 (16.07) \\
\hline \multicolumn{6}{|l|}{ Smoking status ${ }^{\mathrm{b}}$} \\
\hline Never & 46.2 & 66.1 & 43.2 & 50.7 & $52(16.8)$ \\
\hline Ex-smoker & 41.1 & 63.69 & 37.5 & 46.05 & $64.46(14.7)$ \\
\hline Never-smoker & 38.9 & 53.78 & 42.1 & 45 & $65.67(14.9)$ \\
\hline
\end{tabular}

Notes: a'Spearman's rank correlation coefficient; ' Kruskal-Wallis test.

Abbreviations: SGRQ, St George's Respiratory Questionnaire; WHOQOL-BREF, World Health Organization's quality of life abbreviated questionnaire. 
Table 7 Correlation of clinical disease severity indices (illness duration, CLD, MRC grade for dyspnea) and comorbid illness with WHOQOL-BREF and SGRQ

\begin{tabular}{|c|c|c|c|c|c|c|c|c|c|c|}
\hline \multirow[t]{4}{*}{ Characteristics } & \multicolumn{10}{|c|}{ Health-related quality of life score } \\
\hline & \multirow{2}{*}{\multicolumn{2}{|c|}{$\begin{array}{l}\text { Total score } \\
\text { WHOQOL-BREF }\end{array}$}} & \multicolumn{8}{|l|}{ SGRQ } \\
\hline & & & \multicolumn{2}{|c|}{ Symptoms } & \multicolumn{2}{|l|}{ Activity } & \multicolumn{2}{|c|}{ Impacts } & \multicolumn{2}{|c|}{ Total score } \\
\hline & r-value & $P$-value & r-value & $P$-value & r-value & $P$-value & $r$-value & $P$-value & $r$-value & $P$-value \\
\hline Illness duration ${ }^{\mathrm{a}}$ & -0.22 & $0.017 *$ & 0.039 & 0.679 & 0.07 & 0.492 & 0.13 & 0.69 & 0.12 & 0.363 \\
\hline $\mathrm{CLD}^{\mathrm{a}}$ & -0.28 & $0.003 * *$ & 0.57 & $<0.00 I^{* * *}$ & 0.52 & $<0.00$ I*** & 0.41 & $<0.00 I^{* * *}$ & 0.66 & $0.00 I^{* * * *}$ \\
\hline MRC grade for dyspnea ${ }^{a}$ & -0.51 & $<0.00 I^{* * * *}$ & 0.37 & $<0.00$ I*** & 0.60 & $<0.001 * * *$ & 0.55 & $<0.00$ I*** & 0.7 & $<0.001 * *$ \\
\hline Comorbid condition ${ }^{\mathrm{b}}$ & -0.14 & 0.167 & -0.15 & 0.146 & -0.03 & 0.778 & -0.07 & 0.474 & -0.08 & 0.436 \\
\hline
\end{tabular}

Notes: aSpearman's rank correlation coefficient; 'Kruskal-Wallis test; $* P<0.05$; **P $<0.0$ I; $* * * P<0.00$ I.

Abbreviations: CLD, Chronic Lung Disease Severity Index; MRC, Medical Research Council; SGRQ, St George's Respiratory Questionnaire; WHOQOL-BREF, World Health Organization's quality of life abbreviated questionnaire.

to be similarly impaired by the reduced $\mathrm{FEV}_{1}$. There were similar findings when results of the 6-minute walk, an objective measurement of lung and overall fitness, were analyzed. The shorter the distance a patient could walk in 6 minutes, the worse perception of his/her QOL.

In previously reported studies, older age, presence of comorbid illness, less education, and greater smoking consumption resulted in lower QOL. ${ }^{10,11,13-15}$ Surprisingly, no worsening HRQOL measurement correlated significantly with these variables studied in our group of Indians with COPD. The cause for this difference in findings in our patients compared with patients previously reported is not apparent from our data. It may result from small sample size, or it may be related to a unique aspect of the Indian culture regarding aging and age expectations, appreciation for education, or social structure. Ninety-seven percent of the patients studied here were married, and the rest were widowed/widowers. This reflects the very low overall divorce rate in India, and it may be that spousal and societal support may contribute in a significant way to improved QOL. This is an area that warrants further study.

Limitations of this study include the small sample size of 58 patients and the fact that 54 of the 58 subjects were male. The latter may be explained by the fact that tobacco smoking is almost exclusively confined to the male population in India. Also, nine of the original group of 73 were excluded because they were unable to participate in the 6-minute walk, and this may have excluded some patients with more severe disease. However, because this study was performed in a pulmonary diseases clinic, individuals with more severe disease were likely to have been studied. Finally, none of the subjects was on long-term oxygen treatment. This is probably because oxygen concentrators, which have to be paid for by the individuals in the present health care scenario, are not easily available and are prohibitively expensive. Thus, COPD patients who are older and have generally exhausted their resources paying for medical care over the years are unlikely to be in a position to afford this treatment.

\section{Conclusion}

Indian patients with COPD show significantly reduced QOL as measured by standardized questionnaires the WHOQOL-BREF and the SGRQ, similar to COPD patients in other countries. All domains measured, including physical, psychological, social relationships, environmental, symptoms, activity, and impacts, were impaired. Severity of symptoms, duration of illness, and level of dyspnea also influenced the QOL for these individuals. Because COPD is

Table 8 Correlation of spirometry and 6-minute walk with WHOQOL-BREF and SGRQ

\begin{tabular}{|c|c|c|c|c|c|c|c|c|c|c|}
\hline \multirow[t]{4}{*}{ Characteristic } & \multicolumn{10}{|c|}{ Health-related quality of life scores } \\
\hline & \multirow{2}{*}{\multicolumn{2}{|c|}{$\begin{array}{l}\text { Total score } \\
\text { WHOQOL-BREF }\end{array}$}} & \multicolumn{8}{|l|}{ SGRQ } \\
\hline & & & \multicolumn{2}{|c|}{ Symptoms } & \multicolumn{2}{|l|}{ Activity } & \multicolumn{2}{|l|}{ Impacts } & \multicolumn{2}{|c|}{ Total score } \\
\hline & r-value & $P$-value & $r$-value & $P$-value & $r$-value & $P$-value & $r$-value & $P$-value & $r$-value & $P$-value \\
\hline Lung function $\left(\mathrm{FEV}_{1}\right)$ & 0.35 & $0.001 * * *$ & -0.27 & $0.015^{*}$ & -0.39 & $0.00 I^{* *}$ & -0.36 & $0.001 * *$ & -0.39 & $<0.00 I^{* * *}$ \\
\hline $\mathrm{FEV}_{1} / \mathrm{FVC} \%$ & 0.22 & $0.018^{*}$ & -0.18 & 0.053 & -0.30 & $0.002^{* *}$ & -0.17 & 0.063 & -0.23 & $0.013 *$ \\
\hline 6-minute walk test & 0.36 & $<0.00 I^{* * *}$ & -0.06 & 0.501 & -0.28 & $0.003^{* *}$ & -0.27 & $0.003 * *$ & -0.26 & $0.005^{* *}$ \\
\hline
\end{tabular}

Notes: $* p<0.05 ; * * p<0.01 ; * * * p<0.001$.

Abbreviations: FEV , forced expiratory volume in I second; FVC, forced vital capacity; SGRQ, St George's Respiratory Questionnaire; WHOQOL-BREF, World Health Organization's quality of life abbreviated questionnaire. 
an incurable slowly progressive chronic illness, and because only minimal improvement in actual lung function and $\mathrm{FEV}_{1}$ can occur, concentrating therapy on selected potentially more treatable aspects of QOL may result in more effective care.

Further studies should be considered to evaluate the impact of social support, family support, and pulmonary rehabilitation programs on the QOL of COPD patients in India. Also, in view of the increased prevalence of biomass fuel for cooking and heating in rural Indian households, studies need to be conducted to study these effects on patients, particularly women and their QOL.

\section{Acknowledgments}

Ms AS Seema for design, supervision, data analysis, and manuscript; Ms Punitha Ezhilarasu and Ms Jebamani Augustine for supervision and data analysis; Dr Joel J Bechtel for data analysis and manuscript; Dr Devasahayam J Christopher for concept, design, supervision, data analysis, and manuscript; and Ms Sangeetha Raviraj for secretarial assistance.

\section{Disclosure}

The authors report no conflicts of interest in this work.

\section{References}

1. Bethsda MD. National Heart, Lung, and Blood Institute. Global Initiative for Chronic Obstructive Lung Disease. March 2001 (updated 2003) NIH publication 2701A. Available at: http://www.goldcopd.org. Accessed February 2, 2012.

2. Murray CJ, Lopez AD. Evidence based health policy - lessons from the Global Burden of Disease Study. Science. 1996;274(5288):740-743.
3. Chan-Yeung M, Ait-Khaled N, White N, Tan WC. The burden and impact of COPD in Asia and Africa. Int J Tuberc Lung Dis. 2004;8(1): 2-14.

4. World Health Organization. WHO Quality of Life-BREF (WHO QOL-BREF). Available at: http://www.who.int/substance_abuse/ research_tools/whoqolbref/en/. Accessed on May 10, 2004.

5. Jones PW, Spencer S. St George's Respiratory Questionnaire Manual. London, UK: St Georges Hospital Medical School; 2003.

6. Gardner RM. Standardization of spirometry: a summary of recommendations from the American Thoracic Society. The 1987 update. Ann Intern Med. 1998;108(2):217-220.

7. Selim AJ, Ren XS, Fincke G, Rogers W, Lee A, Kazis L. A symptombased measure of the severity of chronic lung disease: results from the Veterans Health Study. Chest. 1997;111:1607-1614.

8. Graydon JE, Ross E. Influence of symptoms, lung function, mood and social support in level of functioning of patients with COPD. Res Nurs Health. 1995; 18(6):525-533.

9. Demir G, Akkoca O, Dogan R, Saryal S, Karabiyikoglu G. The evaluation of dyspnea and quality of life in COPD. Tuberk Toraks. 2003;51(4):365-372.

10. Ferrer M, Alonso J, Morera J, et al. Chronic obstructive pulmonary disease stage and health-related quality of life: the Quality of Life of Chronic Obstructive Pulmonary Disease Study Group. Ann Intern Med. 1997;127(12):1072-1079.

11. Prigatano GP, Wright EC, Levin D. Quality of life and its predictors in patients with mild hypoxemia and chronic obstructive pulmonary disease. Arch Intern Med. 1984;144:1613-1619.

12. Andenaes R, Kalfoss MH, Wahl A. Psychological distress and quality of life in hospitalized patients with chronic obstructive pulmonary disease. J Adv Nurs. 2004;46(5):523.

13. Wijnhoven AH, Kriegsman MW, Hesselink AE, Penninx WJ, de Haan M. Determinants of different dimensions of disease severity in asthma and COPD-pulmonary function and health related quality of life. Chest. 2001;119(4):1034-1042.

14. Anderson KL. The effect of chronic obstructive pulmonary disease on quality of life. Res Nurs Health. 1995;18:547-556.

15. Stewart AL, Greenfield S, Hays RD, et al. Functional status and wellbeing of patients with chronic conditions. Results from the Medical Outcomes Study. JAMA. 1989;262(18):907-913.
International Journal of COPD

\section{Publish your work in this journal}

The International Journal of COPD is an international, peer-reviewed journal of therapeutics and pharmacology focusing on concise rapid reporting of clinical studies and reviews in COPD. Special focus is given to the pathophysiological processes underlying the disease, intervention programs, patient focused education, and self management protocols.

\section{Dovepress}

This journal is indexed on PubMed Central, MedLine and CAS. The manuscript management system is completely online and includes a very quick and fair peer-review system, which is all easy to use. Visit $\mathrm{http}: / / \mathrm{www}$.dovepress.com/testimonials.php to read real quotes from published authors. 